\title{
Higgs boson mass and complex sneutrino dark matter in the supersymmetric inverse seesaw models
}

\author{
Jun Guo, ${ }^{a}$ Zhaofeng Kang, ${ }^{b}$ Tianjun Li ${ }^{a, c}$ and Yandong Liu ${ }^{a}$ \\ ${ }^{a}$ State Key Laboratory of Theoretical Physics and \\ Kavli Institute for Theoretical Physics China (KITPC), \\ Institute of Theoretical Physics, Chinese Academy of Sciences, \\ Beijing 100190, P.R. China \\ ${ }^{b}$ Center for High-Energy Physics, Peking University, \\ Beijing, 100871, P.R. China \\ ${ }^{c}$ School of Physical Electronics, University of Electronic Science and Technology of China, \\ Chengdu 610054, P.R. China \\ E-mail: hustgj@itp.ac.cn, zhaofengkang@gmail.com, tli@itp.ac.cn, \\ ydliu@itp.ac.cn
}

ABstract: The discovery of a relatively heavy Standard Model (SM)-like Higgs boson challenges naturalness of the minimal supersymmetric standard model (MSSM) from both Higgs and dark matter (DM) sectors. We study these two aspects in the MSSM extended by the low-scale inverse seesaw mechanism. Firstly, it admits a sizable radiative contribution to the Higgs boson mass $m_{h}$, up to $\sim 4 \mathrm{GeV}$ in the case of an IR-fixed point of the coupling $Y_{\nu} L H_{u} \nu^{c}$ and a large sneutrino mixing. Secondly, the lightest sneutrino, highly complex as expected, is a viable thermal DM candidate. Owing to the correct DM relic density and the XENON100 experimental constraints, two scenarios survive: a Higgs-portal complex DM with mass lying around the Higgs pole or above $W$ threshold, and a coannihilating DM with slim prospect of detection. Given an extra family of sneutrinos, both scenarios naturally work when we attempt to suppress the DM left-handed sneutrino component, confronting with enhancing $m_{h}$.

KEYWORDS: Supersymmetry Phenomenology

ARXIV EPRINT: 1311.3497 


\section{Contents}

1 Introduction and motivations 1

2 The lightest CP-even Higgs boson mass 2

2.1 The ISS Model with an IR-fixed point 3

2.2 Stau-sterile neutrino contribution to the Higgs boson mass 4

3 A complex sneutrino LSP in the ISS-MSSM $\quad 8$

$\begin{array}{llr}3.1 \text { General simplifications due to complexity } & 9\end{array}$

3.2 The sneutrino LSP confronting with enhancing Higgs boson mass 11

3.2.1 Higgs-portal sneutrino DM inspired by natural SUSY 11

3.2.2 Coannihilating sneutrino LSP inspired by the semi-constrained ISS 12

4 Discussions and conclusion $\quad 14$

\section{Introduction and motivations}

The CMS and ATLAS Collaborations discovered a new resonance around $125.5 \mathrm{GeV}[1,2]$. From the latest full collected data announced at the Moriond 2013 conference, it is quite Standard Model (SM)-like. If this is confirmed at the next run of the $\sqrt{s}=14 \mathrm{TeV} \mathrm{LHC}$, it would complete the picture of SM. But we can never conclude that the discovery of a highly SM-like Higgs boson at the LHC indicates an end to the particle physics: on the theoretical side, the SM suffers the notorious gauge hierarchy problem if the discovered resonance is indeed a fundamental spin-0 boson; on the phenomenological side, the SM can not explain the tiny neutrino mass origin and has no candidate for dark matter (DM), both of which are clear signals for new physics beyond the SM.

Supersymmetry (SUSY) is still the most promising underlying theory to account for these two sides simultaneously. The supersymmetric SMs (SSMs) are free of quadratic divergences involving scalars, and provide a weakly interactive massive particle (WIMP) DM candidate if $R$-pariy is conserved, i.e., the Lightest Supersymmetric Particle (LSP) such as the lightest neutralino [3]. Of course, to explain the neutrino masses and mixings, we may have to supersymmetrize the well studied models with seesaw mechanisms. Among them, the inverse seesaw (ISS) mechanism $[4,5]$ has an obvious advantage: it is suited for the TeV-scale seesaw mechanism without turning to tiny Yukawa couplings between the neutrinos and Higgs doublet: $Y_{\nu} L H_{u} \nu^{c}$. This property is found to be capable of mitigating the great stress in the Minimal SSM (MSSM) which, to have the relatively heavy SM-like Higgs boson mass, incurs a rather serious fine-tuning from generating both the weak scale and LSP neutralino dark matter phenomenology [6-11]. 
To demonstrate the consequence of this property, we consider that the new (single family of) Yukawa coupling develops an IR-fixed point, which predicts $Y_{\nu} \simeq 0.75$. This new large Yukawa coupling involving $H_{u}$ at the low energy contributes to the lightest CPeven Higgs boson mass $m_{h}$ radiatively. Using the effective potential method $[12,13]$, we first analytically calculate such corrections in some simplified cases and then employ the full numerical analyses. Enhancement up to $4 \mathrm{GeV}$ can be obtained in the case of a large sneutrino mixing. This helps to alleviate the tension between a relatively heavy SM-like Higgs boson and the weak-scale naturalness.

The sneutrino LSP in the SSMs with low-scale seesaw mechanism may be a good alternative of the neutralino LSP DM [14-17], especially after the discovery of the SMlike Higgs boson and null results from the DM detection experiments like XENON100 [18]. Specified to the low scale supersymmetric ISS, the sneutrino LSP is expected to be complex. This restricts the sneutrino DM into two possibilities: (I) Essentially it belongs to the Higgs-portal complex DM, and its mass has to be around $m_{h} / 2$ or above the $W$ boson mass $m_{W}$; (II) It is a coannihilating DM, for example, the sneutrino LSP and Higgsino coannihilation. This allows a rather weak coupling between sneutrino DM and visible particles, so it is hard to be detected. Given an extra family of sneutrinos, both scenarios naturally work when we attempt to suppress the left-handed sneutrino DM component, confronting with enhancing $m_{h}$.

This paper is organized as follows. In section 2, we briefly introduce the model and then calculate the radiative correction to Higgs boson mass from the neutrino Yukawa coupling at the IR-fixed point. In section 3, we discuss the complex sneutrino dark matter phenomenology and investigate how they are consistent with the requirement of enhancing Higgs boson mass. The section 4 includes discussions and conclusion.

\section{The lightest CP-even Higgs boson mass}

The models equipped with a low scale seesaw mechanism receive special attentions, by virtue of its potential to be tested within our near future experiments. Specified to the SSMs, after the discovery of a relatively heavy SM-like Higgs boson, models capable of enhancing the Higgs boson mass $m_{h}$ gain further theoretical preference, as stressed in the introduction. In type-I and III seesaw mechanisms, where the small neutrino mass $m_{\nu} \sim Y_{\nu}^{2} v^{2} / M_{R}$ with $M_{R}$ the seesaw scale, the enhancement is impossible [20] because a low scale $M_{R}$ is at the price of a negligibly small $Y_{\nu}{ }^{1}$ By contrast, in the type-II and inverse seesaw mechanisms the smallness of $m_{\nu}$ has other origins, and then the Higgs doublets are allowed to have large neutrino Yukawa couplings. For instance, the supersymmetric type-II seesaw mechanism can even enhance $m_{h}$ at the tree level [22] (actually, it can simultaneously enhance the di-photon rate [22]). However, such models are difficult to be embedded into a pertubative Grand Unified Theory (GUT) picture. The inverse seesaw models, where only

\footnotetext{
${ }^{1}$ In ref. [21], it was pointed out that in type-I seesaw the correction to Higgs boson mass can be significant. For a high scale $M_{R}$ (thus allowing $Y_{\nu} \sim 1$ ) but at the same time a very large soft mass squared for the right-handed sneutrino, unfortunately, the correction is negative.
} 
singlets are involved, are potential to lift the Higgs boson mass (at one-loop level) without violating GUT.

In this section we will first briefly review the minimal supersymmetric ISS mechanism, and then discuss one of the new Yukawa couplings with the IR-fixed point behavior and its implication to the correction on the SM-like Higgs boson mass.

\subsection{The ISS Model with an IR-fixed point}

The minimal supersymmetric model with ISS is the MSSM extended by two extra singlets $\nu^{c}$ and $N$ (a single family for the time being), which carry lepton numbers -1 and 1 and are dubbed as right-handed neutrino (RHN) and Dirac partner RHN (DRHN), respectively. The superpotential, asides from the ordinary MSSM terms, can be written as a sum of terms respecting the lepton number and a term violating it explicitly but slightly:

$$
W_{I S S}=\left(Y_{\nu} \nu^{c} L H_{u}+M_{R} \nu^{c} N\right)+\frac{1}{2} \mu_{N} N^{2} .
$$

The model contains one dimensionless parameter $Y_{\nu}$, and two dimension-one mass parameter $M_{R}$ and $\mu_{N}$. The $\mu_{N}$ term softly breaks the lepton number by two units and largely accounts for the smallness of tiny neutrino mass. ${ }^{2}$

The model naturally gives rise to a low scale seesaw mechanism without turning to small parameters except for the massive parameter $\mu_{N}$. In the basis $\left(\nu, \nu^{c}, N\right)$ the neutrino mass matrix is given by

$$
\mathcal{M}_{F}=\left(\begin{array}{ccc}
0 & Y_{\nu} v_{u} & 0 \\
Y_{\nu} v_{u} & 0 & M_{R} \\
0 & M_{R} & \mu_{N}
\end{array}\right)
$$

where $v_{u}$ is the Vacuum Expectation Value (VEV) of Higgs field $H_{u}$, i.e., $\left\langle H_{u}\right\rangle=v_{u}$. This matrix leads to the following combination as the light Majorana neutrino

$$
\nu_{1} \approx \sin \theta_{1} \nu_{L}-\cos \theta_{1} \nu^{c}
$$

where the mixing angle is approximated to be $\sin \theta_{1} \approx M_{R} / \sqrt{m_{D}^{2}+M_{R}^{2}}$ with $m_{D} \equiv Y_{\nu} v_{u}$ the Dirac mass for $\nu_{L}$ and $\nu^{c}$. The neutrino mass assumes a form of

$$
m_{\nu} \simeq \frac{m_{D}^{2}}{M_{R}^{2}} \mu_{N}
$$

Notably, if $\mu_{N}$, for some reason, can be arbitrarily small, then the sub-eV neutrino mass scale can be obtained without turning to the large suppression from extremely small $Y_{\nu}$ or (and) large $M_{R}$. This merit of the ISS model is the basic observation of our article. But note that owing to the non-unitary constraint, $M_{R}$ should be several times larger than $m_{D}$. From ref. $[23,24]$ we set a rough bound

$$
M_{R} \gtrsim 10 m_{D}
$$

\footnotetext{
${ }^{2}$ Based on the next-to-MSSM, the Dirac mass $M_{R}$ can be dynamically generated [35, 53]. In particular, ref. [35] observed that, in the presence of a singlet with a TeV-scale VEV, the small $\mu_{N}$-term can be related to a dimension-five operator suppressed by the Planck scale.
} 


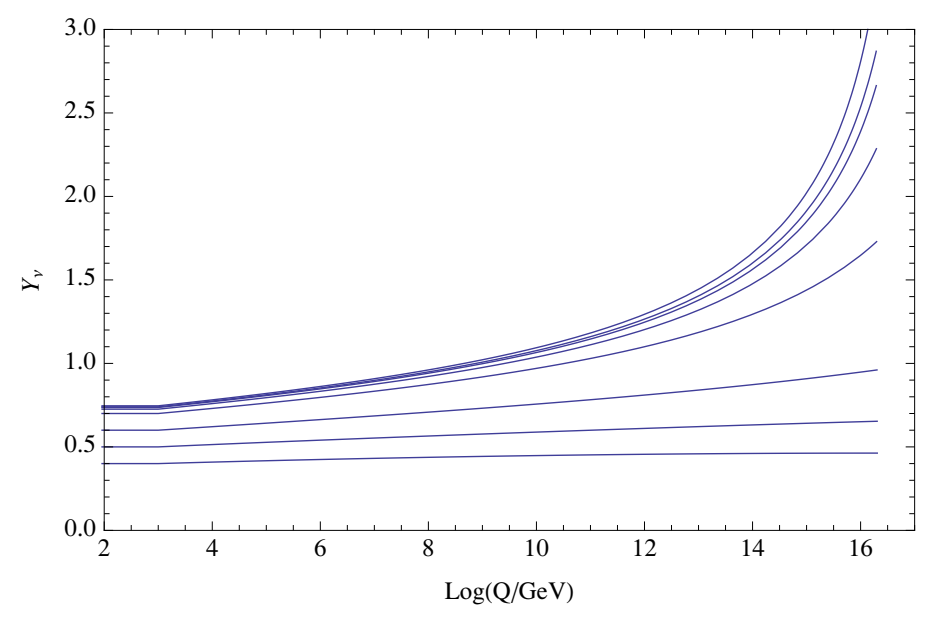

Figure 1. Renormalization group equation running trajectory for $Y_{\nu}$ in the inverse seesaw model, showing that the Yukawa coupling has an infrared-stable quasi-fixed point for large $Y_{\nu}$. Here, $m_{t}=173.1$ and $\tan \beta=10$ are chosen.

so as to make $\theta_{1} \simeq \pi / 2$, i.e., the neutrino is dominated by left-handed neutrino. Finally, $\nu^{c}$ and $N$ form a Dirac fermion with mass approximately given by

$$
M_{R}+m_{D}^{2} / 2 M_{R}
$$

For a reason discussed later, we are interested in how large $Y_{\nu}$ is allowed by pertubativity up to the GUT scale. Interestingly, we find that there is an IR-fixed point structure predicting $Y_{\nu} \lesssim 0.75$. We present the Yukawa running in this model and focus on the new Yukawa term which is illustrated in figure 1. For numerical calculation, we choose the SUSY-breaking scale $m_{S}=800 \mathrm{GeV}$, new sterile neutrino scale $M_{R}=1000 \mathrm{GeV}$, and we simply consider one generation new sterile neutrinos. The new Yukawa coupling has infrared quasi-fixed point behavior, which restrict how large it can be at the TeV-scale while maintaining consistent with perturbative unification. Here for fixed-point trajectory we adopt the same definition like in [25] which request the new Yukawa couplings is less than or equal to 3 .

\subsection{Stau-sterile neutrino contribution to the Higgs boson mass}

It is well known that at tree level the SM-like Higgs boson mass $m_{h}$ is predicted to be lighter than $M_{Z}$ in the MSSM. This necessitates an significant radiative correction from the stoptop sector to lift the Higgs boson mass near $125 \mathrm{GeV}$, which is discovered by the recent CMS and ATLAS Collaborations [1, 2]. However, such large correction typically requires stops at the TeV scale and then renders the MSSM highly fine-tuned [6]. Therefore, how to lift the Higgs boson mass at the less price of fine-tuning is a very interesting question.

The supersymmetric ISS model with the IR-fixed point just provides a new source of enhancement via the stau-sterile neutrino correction. We calculate the corrections following the effective potential method $[12,13]$ from the Colenman-Weinberg potential [26] of the 
Higgs field via a general formula

$$
\begin{aligned}
\Delta V\left(H_{u}\right) & =2 \sum_{i}\left[F\left(M_{S_{i}}^{2}\right)-F\left(M_{F_{i}}^{2}\right)\right], \\
F\left(M^{2}\right) & =\frac{M^{4}}{64 \pi^{2}}\left[\ln \left(M^{2} / Q^{2}\right)-3 / 2\right],
\end{aligned}
$$

with $Q$ the renormalization scale. Index $i$ runs over all the scalar and fermion couplings to Higgs doublets. Restricted to the neutrino sector, the fermion spectrum contains a light Majorana neutrino, whose contribution to the effective potential is proportional to $\left(\mu_{N} / M_{R}\right)^{4}$ and thus can be safely dropped. While the Dirac sterile neutrinos have a large mass given by eq. (2.6). We postpone the discussion on the scalar spectrum to the next paragraph. Now, in the decoupling region, i.e., $m_{A^{0}}^{2} \gg m_{h}^{2}$, the effective potential gives the following correction to $m_{h}^{2}$

$$
\Delta m_{h}^{2}=\left\{\frac{\sin ^{2} \beta}{2}\left[\frac{\partial^{2}}{\partial v_{u}^{2}}-\frac{1}{v_{u}} \frac{\partial}{\partial v_{u}}\right]+\frac{\cos ^{2} \beta}{2}\left[\frac{\partial^{2}}{\partial v_{d}^{2}}-\frac{1}{v_{d}} \frac{\partial}{\partial v_{d}}\right]+\sin \beta \cos \beta \frac{\partial^{2}}{\partial v_{u} \partial v_{d}}\right\} \Delta V .
$$

We now turn our attention to the scalar spectrum. First, the supersymmetric ISS model introduces new soft terms

$$
-\mathcal{L}_{\text {soft }}=-\mathcal{L}_{\text {soft }}^{\mathrm{MSSM}}+m_{\widetilde{\nu}^{c}}^{2}\left|\tilde{\nu}^{c}\right|^{2}+m_{\widetilde{N}}^{2}|\tilde{N}|^{2}+\left(Y_{\nu} A_{Y_{\nu}} \tilde{L} \tilde{\nu}^{c} H_{u}+B_{M_{R}} \tilde{\nu}^{c} \tilde{N}+\frac{1}{2} B_{\mu_{N}} \tilde{N}^{2}+\text { c.c. }\right),
$$

where $\mathcal{L}_{\text {soft }}^{\mathrm{MSSM}}$ is the MSSM SUSY breaking soft term. $B_{M_{R}} \sim m_{3 / 2} M_{R}$ with $m_{3 / 2}$ the gravitino mass measuring the scale of soft breaking parameters. It is noticed that in our scenario the lepton number violating soft breaking parameter $B_{\mu_{N}} \sim m_{3 / 2} \mu_{N}$ is irrelevantly small (we will come back to this point later), and consequently three sneutrinos are highly complex scalars. Now, in the basis $\left(\widetilde{\nu}_{L},\left(\widetilde{\nu}^{c}\right)^{*}, \widetilde{N}\right)$ the sneutrino mass squared matrix takes a form of

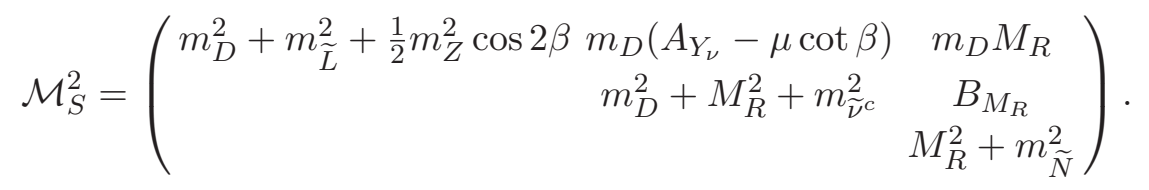

Because the analytical eigenvalues are extremely lengthy, in the ensuing discussion we will consider some solvable limits to demonstrate which parameters can lift the Higgs boson mass.

There are two mixing terms which depend on $H_{u}$ and thus contribute to $m_{h}^{2}$ in terms of eq. (2.8), one is the soft trilinear term $X_{\nu}=A_{Y_{\nu}} v_{u}-\mu \cot \beta$, while the other one comes from the F-term of $\nu^{c}$, i.e., $Y_{\nu} v_{u} M_{R}=m_{D} M_{R}$ (In light of the effective potential method, any origin of correction to $m_{h}$ can be traced back to the matrix entries depending on $m_{D}$ ). Turning off the mixing $A$-term will lead to a solvable matrix. As a warm up, we first consider the case by turning off the $A_{\nu}$ and $B_{M_{R}}$ mixing terms, setting the soft SUSY-breaking mass squares equal to $m_{S}^{2}$ and neglecting the small electroweak D-term contribution. Then in the mass eigenstates the three sneutrino mass squares are given by

$$
m_{S}^{2}, \quad m_{D}^{2}+M_{R}^{2}+m_{S}^{2}, \quad m_{D}^{2}+M_{R}^{2}+m_{S}^{2} .
$$


The eigenstate corresponding to $m_{S}^{2}$, which is independent on $H_{u}$, does not contribute to the effective potential. But it is merely a result of the approximations which we have taken. Using eq. (2.8) we get

$$
\Delta m_{h}^{2}=\frac{1}{4 \pi^{2}} \sin ^{2} \beta Y_{\nu}^{2} m_{D}^{2} \ln \frac{M_{R}^{2}+m_{S}^{2}}{M_{R}^{2}} .
$$

It is similar to the non-mixing stop case but quantitatively less important due to a color factor and the smaller Yukawa coupling which leads to a suppression $\left(Y_{\nu} / h_{t}\right)^{4}$. Even worse is that, from the neutrino side we have $M_{R} \sim 1 \mathrm{TeV}$ and consequently here the logarithmic enhancement is rather limited if $m_{S}$ is constrained around the TeV scale by naturalness. Thus, the correction from sneutrino is less than 10 percents of that from the stop case, see the left panel of figure 3 .

Next we take into account the soft trilinear term $X_{\nu}$, which would bring much difference in lifting $m_{h}$. If other approximations are the same as the non-mixing case, we are still able to find an analytical expression for the correction, in spite of somewhat complication

$$
\begin{aligned}
\Delta m_{h}^{2}=\frac{1}{4 \pi^{2}} \sin ^{2} \beta Y_{\nu}^{2} m_{D}^{2} & \left(\ln \frac{m_{S}^{2}+M_{R}^{2}}{M_{R}^{2}}+\frac{X_{\nu}^{4}+2 X_{\nu}^{2} M_{R}^{2}}{M_{R}^{4}}\right. \\
& \left.-\frac{2 X_{\nu}^{4} m_{S}^{2}+X_{\nu}^{4} M_{R}^{2}+4 X_{\nu}^{2} m_{S}^{2} M_{R}^{2}}{2 M_{R}^{6}} \ln \frac{m_{S}^{2}+M_{R}^{2}}{m_{S}^{2}}\right) .
\end{aligned}
$$

We would like to comment on the origins of various terms in the above equation. ${ }^{3}$ The first logarithmic term exactly reproduces the result given in eq. (2.12), extracting the correction to the Higgs quartic coupling $\lambda_{h}$, which is encoded in the Renormalization Group Equation (RGE) running from the supersymmetry breaking scale defined by the heavy sneutrino mass scale to the Dirac sterile neutrino mass scale $M_{R}$. While the second term stands for the mixing effect after integrating out the sneutrinos, included as a shift to the boundary $\lambda_{h}$ (In the explicit Feymann diagram calculations, it can be obtained from the triangle and box diagrams.). The last term has an obscure dependence on the logarithmic $\log \left(\left(m_{S}^{2}+M_{R}^{2}\right) / m_{S}^{2}\right)$, stemming from the different mass scales of $\widetilde{\nu}_{L}$ and $\widetilde{\nu}^{c}$ as shown in eq. (2.10). Such a hybrid of the mixing and logarithmic terms is absent in the stop system, and noticeably it is negative.

We now quantitatively analyze the corrections given in eq. (2.13). $m_{D}$ has been fixed by virtue of the IR-fixed of $Y_{\nu}$, and then it is not difficult to find that $\Delta m_{h}^{2}$ actually depends on two dimensionless parameters, $x_{s}=m_{S}^{2} / M_{R}^{2}$ and $x_{a}=X_{\nu}^{2} / M_{R}^{2}$. Denoting the function in the brackets as $f\left(x_{s}, x_{a}\right)$, it consists of three parts: the first and second parts are positive while the third part is negative. Hence the maximal mixing scenario here is more subtle than the stop case. It is illustrative to consider two limits of $x_{s}$ : (1) If $x_{s} \gg 1$, i.e., the large SUSY breaking soft mass terms, we have a simple expression for $f\left(x_{s}, x_{a}\right) \rightarrow \log x_{s}-x_{a}^{2} / 2 x_{s}$. Thereby the mixing effect is negative but suppressed by large $x_{s}$. (2) Oppositely, if $x_{s} \ll 1$ we have $f\left(x_{s}, x_{a}\right) \rightarrow x_{a}^{2}\left(1+\log \sqrt{x_{s}}\right)+2 x_{a}$. Thus, a moderate

\footnotetext{
${ }^{3}$ We note that our approximation expression is different from that in ref. [53] which is consistent with that in ref. [25]. However, from our understanding, the particle contents and interactions in their inverse seesaw models are not the same as the extended MSSM with extra vector-like particles.
} 


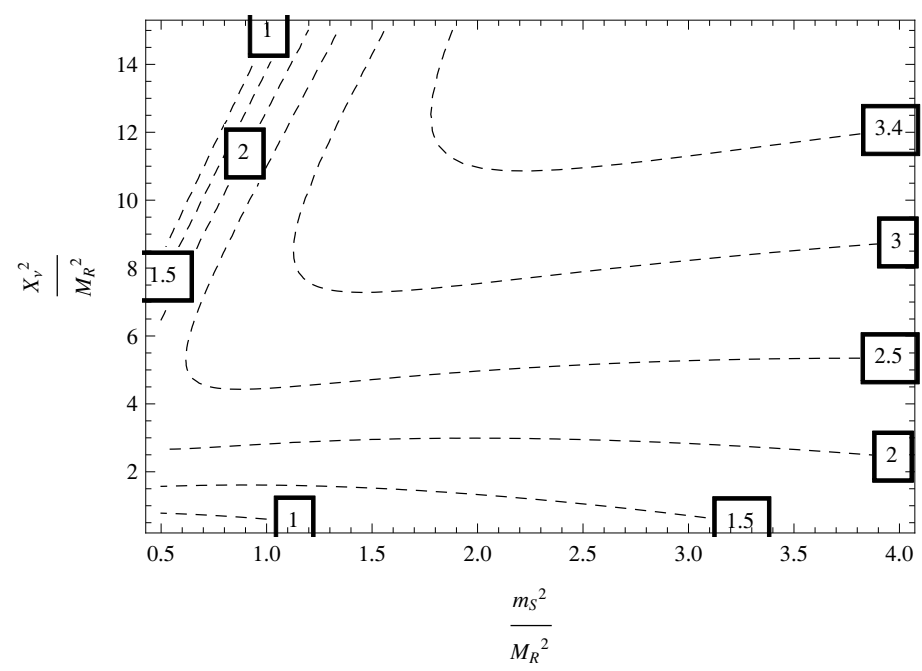

Figure 2. $\frac{X_{\nu}^{2}}{M_{R}^{2}}$ versus $\frac{m_{S}^{2}}{M_{R}^{2}}$ for the contour of $\Delta m_{h}$, which is defined as $\sqrt{(123 \mathrm{GeV})^{2}+\Delta m_{h}^{2}}-$ $123 \mathrm{GeV}$ throughout this work.
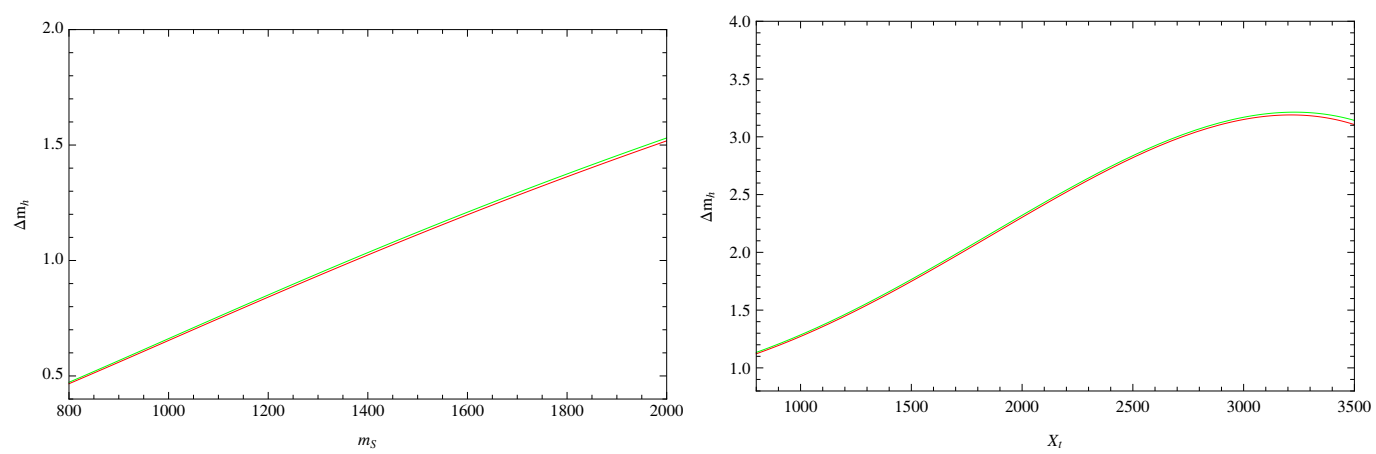

Figure 3. Left panel: $\Delta m_{h}$ versus $m_{S}$ with vanishing soft trilinear term. Right panel: $\Delta m_{h}$ versus $X_{\nu}$ for $M_{R}=1000 \mathrm{GeV}, m_{S}=800 \mathrm{GeV}$, and $\tan \beta=15$. The red lines correspond to the full numerical results and green lines the approximated results.

$x_{s}$ is needed to maximize the corrections. Explicitly, we present a contour plot of $\Delta m_{h}$ in figure 2.

We examine the difference between the approximately analytical and full numerical treatments. Figure 3 shows that they give rise to almost the identical results, in the trivial case of non-mixing (left panel) and the case with $A_{\nu}$ mixing only (right panel). This indicates that the expression in eq. (2.13) works well for universal soft masses. In figure 3, for simplicity we set $B_{M_{R}} \sim 0$. However, a non-zero $B_{M_{R}}$ may lead to an appreciable change. Although it is still possible to develop an analytical expression for $\Delta m_{h}$, it is too lengthy to convey any useful information. Therefore, we only display the change numerically in figure 4, with color codes of lines as before. It is clearly seen that, as $\left|B_{M_{R}}\right|^{1 / 2}$ approaches the sneutrino mass scale, the discrepancy between the full result and the approximation eq. (2.13) becomes rather significant. 

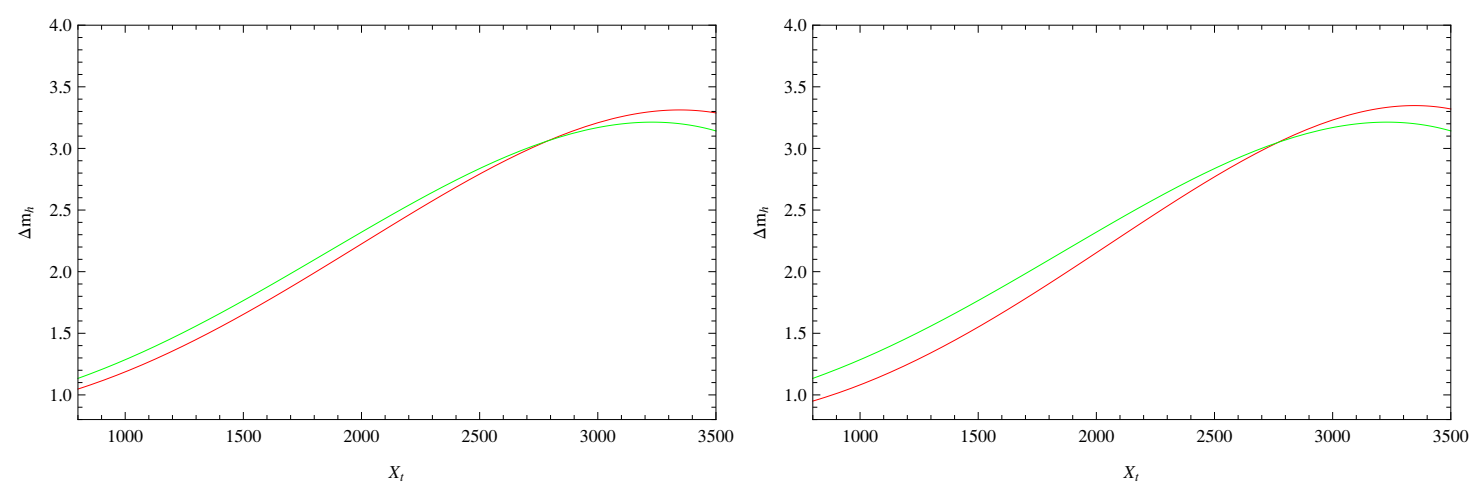

Figure 4. Left: $\Delta m_{h}$ versus $m_{S}$ for the full numerical results with $B_{M_{R}}=0.5 m_{S}^{2}$; right: $\Delta m_{h}$ versus $m_{S}$ for the full numerical results with $B_{M_{R}}=m_{S}^{2}$. We have fixed $M_{R}=1000 \mathrm{GeV}, m_{S}=$ $1200 \mathrm{GeV}$, and $\tan \beta=15$.

\section{A complex sneutrino LSP in the ISS-MSSM}

The presence of a DM candidate, i.e., the neutral LSP, is one of the major attractions of the SSMs. The lightest neutralino receives the most intensive attention. However, the heaviness of the soft spectrum, owing to a relatively heavy Higgs boson and null sparticle searches, along with the stringent bounds from direct detection experiments such as XENON100 [18], ${ }^{4}$ now threaten the viable neutralino DM. Another neutral LSP candidate, the lightest sneutrino, has been investigated by many authors in many contexts $[14-17,27-$ 29], and now may show some advantages.

Roughly speaking, the sneutrino LSPs can be classified into the following three types

Complex sneutrino. As in the MSSM, the left-handed sneutrino $\widetilde{\nu}_{L}$, just the original proposal $[14,15]$, is a complex scalar due to the conservation of global lepton number $\mathrm{U}(1)_{L}$. But the $Z$-boson mediated DM-nucleon spin-independent (SI) scattering has a very large cross section $\sigma_{\mathrm{SI}}[27]$, which excludes the sneutrino LSP in the MSSM. Beyond it, a complex sneutrino LSP dominated by the SM singlets may be realized in the models which conserve the lepton number with a high degree [35-42].

Real sneutrino. Taking into account for generating tiny neutrino masses via the seesaw mechanisms, $\mathrm{U}(1)_{L}$ should be broken and then induces the mass splitting between the CP-odd and even components of complex sneutrino. If splitting is large, we actually have a real sneutrino LSP [28]. Consequently, $\sigma_{\mathrm{SI}}$ from $Z$-boson exchange will be zero.

Pseudo-complex sneutrino. When the splitting is small, which is the usual case because of suppression from small neutrino mass, we will get a pseudo-complex sneu-

\footnotetext{
${ }^{4}$ After the completion of this work, the LUX Collaboration announced their new results, which gave the even more stringent constraint, one magnitude of order stronger than the XENON100 experiment. But this does not qualitatively affect our discussions, so we only mention XENON100 in the text and show LUX data in figures.
} 
trino $[28,30-34]$. In this case, the DM-nucleon scattering mediated by $Z$-boson becomes inelastic and may be kinematically forbidden.

In the low-scale ISS-MSSM, $\mathrm{U}(1)_{L}$ is broken by $\mu_{N} N^{2} / 2$ and the corresponding soft bilinear term $B_{\mu_{N}} \widetilde{N}^{2} / 2=m_{\mathrm{SUSY}} \mu_{N} \widetilde{N}^{2} / 2$. Provided that there is no peculiar SUSY-breaking mediation such as in ref. [36-42], the mass splitting between the CP-even and CP-odd parts of $\widetilde{N}_{1}$, due to the soft bilinear term, is suppressed by the neutrino mass

$$
\delta m \sim \frac{m_{\mathrm{SUSY}} \mu_{N}}{2 m_{\widetilde{N}_{1}}} \sim\left(\frac{m_{\mathrm{SUSY}}}{2 m_{\widetilde{N}_{1}}}\right)\left(\frac{M_{R}^{2}}{m_{D}^{2}}\right) m_{\nu},
$$

which is around $10 \mathrm{eV}$. Thereby, in this model the sneutrino LSP is expected to be complex [35]. Moreover, it is thermal since it is free of extreme suppression from Yukawa couplings with the visible sector. But note that enhancing $\mathrm{U}(1)_{L}$-violation by a abnormally large $B_{\mu_{N}} \sim \mathcal{O}\left(\mathrm{GeV}^{2}\right)$ can lead to an inelastic sneutrino DM, which actually is the case in most references $[33,34,43,44]$. In this article, we insist on a normal SUSY breaking mediation mechanism to account for soft terms, and then we have a (highly) complex sneutrino LSP. Our purpose is to explore the viable sneutrino DM scenarios consistent with the enhancing Higgs boson mass.

\subsection{General simplifications due to complexity}

We investigate what kind of mixture can lead to a good sneutrino DM, which has correct relic density, is allowed by the XENON100 experiment and does not incur tremendous fine-tuning. In general, for the moment we do not restrict discussions to the setup made in the previous section. From the first glance, the sneutrino LSP is complicated because of three complex sneutrino system, but the complexity of the sneutrino LSP greatly simplifies the discussions.

Above all, we have to suppress the left-handed sneutrino fraction. The lightest sneutrino is a superposition of three sneutrinos

$$
\widetilde{N}_{1}=C_{\widetilde{\nu}_{L}} \widetilde{\nu}_{L}+C_{\widetilde{\nu}^{c}}\left(\widetilde{\nu}^{c}\right)^{*}+C_{\widetilde{N}} \widetilde{N}
$$

where the singlet fraction must dominate to suppress the $Z$-mediated DM-nucleon scattering. It is justified to make an estimation on the doublet fraction

$$
C_{\widetilde{\nu}_{L}} \simeq \frac{m_{D} A_{\nu}}{m_{\widetilde{\nu}_{L}}^{2}} \sin \theta_{23}+\frac{m_{D} M_{R}}{m_{\widetilde{\nu}_{L}}^{2}} \cos \theta_{23}
$$

which is quite precise in the case of large mass splitting between $\widetilde{\nu}_{L}$ and other two sneutrinos. $\theta_{23} \in(-\pi / 2,0)$ is the mixing angle of $\widetilde{\nu}^{c}$ and $\widetilde{N}$ from the 23 -submatrix of eq. (2.10)

$$
\tan \theta_{23} \approx P_{23}-\sqrt{P_{23}^{2}+1}, \quad P_{23} \equiv\left(m_{D}^{2}+m_{\widetilde{\nu}^{c}}^{2}-m_{\widetilde{N}}^{2}\right) / 2 B_{M_{R}} .
$$

In eq. (3.2) $C_{\widetilde{\nu}^{c}} \approx-\sin \theta_{23}$ and $C_{\widetilde{N}} \approx-\cos \theta_{23}$. The DM-proton SI scattering cross section is $\sigma_{p}=\mu_{p}^{2} f_{p}^{2} / \pi$ [3], with $\mu_{p}$ the DM-proton reduced mass. For a DM with mass around $100 \mathrm{GeV}$, XENON100 imposes the upper bound $f_{p} \lesssim 0.3 \times 10^{-8} \mathrm{GeV}^{-2}$ and in turn

$$
f_{p}=C_{\widetilde{\nu}_{L}}^{2} g_{2}^{2} / m_{Z}^{2} \lesssim 0.3 \times 10^{-8} \mathrm{GeV}^{-2} \Rightarrow C_{\widetilde{\nu}_{L}} \lesssim 0.01
$$


A natural suppression needs a multi-TeV $\widetilde{\nu}_{L}$ for $Y_{\nu} \sim \mathcal{O}(0.1)$. By contrast, a much lighter $\widetilde{\nu}_{L}$ (but still heavier than $\widetilde{N}$ and $\widetilde{\nu}^{c}$ ) is allowed given a sufficiently small $Y_{\nu}$.

With such a small $C_{\widetilde{\nu}_{L}}$, the dynamics of the sneutrino LSP largely reduces to that of a Higgs-portal complex scalar DM. Concretely, $\widetilde{N}_{1}$ annihilates into SM particles through four ways: (1) Contact interactions with $H_{u}$ from $\left|F_{\nu_{L}}\right|^{2}$; (2) $h$ propagating in the $s$-channel; (3) Higgsinos/sneutrinos propagating in the $t$-channel; (4) Gauge interactions inheriting from $\widetilde{\nu}_{L}$. Decoupling $\widetilde{\nu}_{L}$ means only case (1) left, giving rise to a Higgs-portal complex scalar DM

$$
\left|F_{\nu_{L}}\right|^{2} \rightarrow \lambda_{H}\left|\widetilde{N}_{1}\right|^{2}\left|H_{u}\right|^{2}, \quad \lambda_{H} \equiv\left(\sin \theta_{23} Y_{\nu}\right)^{2}
$$

But cases (2) and (3) may cause deviations from an exact Higgs-port DM. In the first, the Higgsino-mediated processes might be important for light DM well below $m_{W}$, since their cross sections are $\sim \mathcal{O}\left(\lambda_{H}^{2} m_{\widetilde{N}_{1}}^{2} / 32 \pi M_{\text {Higgsino }}^{4}\right)$, with further velocity/helicity suppressions [35]. But owing to the bound indicated in eq. (3.9), we find that it is far from enough to give the correct relic density and thus can be ignored. Next, if $C_{\widetilde{\nu}_{L}}$ is not extremely suppressed, processes involving $\widetilde{\nu}_{L}$ contributions to DM cross sections at higher order of $C_{\widetilde{\nu}_{L}}$ can be enhanced by large massive couplings. This is seen from the following terms, which are absent in the ordinary Higgs-portal DM models,

$$
\begin{aligned}
-\mathcal{L}_{\text {trilinear }} & =\left|F_{\nu^{c}}\right|^{2}+\left(Y_{\nu} A_{\nu} \widetilde{L} H_{u} \widetilde{\nu}^{c}+\text { c.c. }\right) \\
& \rightarrow-\frac{\left(C_{\widetilde{\nu}_{L}} m_{\widetilde{\nu}_{L}}\right)^{2}}{\sqrt{2} v_{u}} h\left|\widetilde{N}_{1}\right|^{2}-C_{\widetilde{\nu}_{L}} \frac{m_{\widetilde{\nu}_{L}}^{2}}{\sqrt{2} v_{u}} h \widetilde{\nu}_{L} \widetilde{N}_{1}^{*}+\text { c.c. }
\end{aligned}
$$

The presence of extra $C_{\widetilde{\nu}_{L}}$ in the first and second terms are traced back to the fact that these trilinear couplings are also sources of the mixing terms between $\widetilde{\nu}_{L}$ and singlets, see eq. (2.10).

We proceed to argue that terms in eq. (3.7) can make no difference. The first term of eq. (3.7) affects processes mediated by $h$. With it, the massive coupling constants of $h\left|\widetilde{N}_{1}\right|^{2}, \mu_{h}$, takes a form of

$$
\mu_{h}=\sqrt{2} v_{u} \lambda_{H}\left[1+\left(C_{\widetilde{\nu}_{L}} m_{\widetilde{\nu}_{L}} / \sqrt{2 \lambda_{H}} v_{u}\right)^{2}\right] .
$$

The second term stands for the deviation from the exact Higgs-portal. In terms of eq. (3.3), its order of magnitude is estimated to be at least $\sim \mathcal{O}\left(A_{\nu} / \sqrt{2} m_{\widetilde{\nu}_{L}}\right)^{2}$, which is reliable barring the large cancellation in eq. (3.3). So, for a relatively large $A_{\nu}$ but small $m_{\widetilde{\nu}_{L}}$, which means an appreciable $C_{\widetilde{\nu}_{L}}$, the trilinear soft term dominates $\mu_{h}$. $\sigma_{\mathrm{SI}}$ from $h$ implies the bounds

$$
\left(C_{\widetilde{\nu}_{L}} m_{\widetilde{\nu}_{L}}\right)^{2} / \sqrt{2} v_{u} m_{\mathrm{DM}}, \sqrt{2} v_{u} \lambda_{H} / m_{\mathrm{DM}} \lesssim \mu_{h} / m_{\mathrm{DM}} \lesssim 0.12 \times\left(\sigma_{p}^{\mathrm{up}} / 10^{-9} \mathrm{pb}\right)^{1 / 2} .
$$

But the dynamics of the Higgs-portal DM with and without deformation (by trilinear soft terms) are the same, provided that annihilation into a pair of $h$ via the contact interaction in eq. (3.6) is irrelevant. Explicitly, it possesses $\sigma_{h h}^{c} v=\left(\sqrt{2} \lambda_{H} v_{u} / m_{\mathrm{DM}}\right)^{2} / 128 \pi v_{u}^{2} \lesssim 0.5$ 
pb, a marginally relevant value. Thus, this deviation does not matter much. And the second term of eq. (3.7) is not important neither. It gives rise to the sneutrino-mediated annihilation into a pair of $h$, with a thermal averaged cross section

$$
\sigma_{h h}^{t} v \simeq \frac{1}{32 \pi}\left(\frac{C_{\widetilde{\nu}_{L}}^{2} m_{\widetilde{\nu}_{L}}^{2}}{\sqrt{2} v_{u} m_{\mathrm{DM}}}\right)^{2} \frac{1}{v_{u}^{2}}
$$

Even if the value in the bracket saturates its upper bound shown in eq. (3.9), this cross section merely gives $1 \mathrm{pb}$. Actually, it can never be saturated for $m_{\widetilde{\nu}_{L}} \lesssim 1 \mathrm{TeV}$ and $m_{\mathrm{DM}}>m_{h}$. In summary, the complex sneutrino DM is reduced to the Higgs-portal DM.

\subsection{The sneutrino LSP confronting with enhancing Higgs boson mass}

With the above analyses, in this subsection we explore the viable scenarios for sneutrino DM, taking into account the requirement to lift the Higgs boson mass. But a single family of sneutrino fails, because of the contradiction between these two aspects. On the one hand, to significantly lift $m_{h}$ we need $Y_{\nu} \sim 1$ and thereby $10 m_{D} \lesssim M_{R} \sim \mathcal{O}(1) \mathrm{TeV}$. On the other hand, it renders sneutrinos heavy. Moreover, the mixings between $\widetilde{\nu}_{L}$ and $\widetilde{\nu}^{c} / \widetilde{N}$ are large by virtue of the large off-diagonal entries, i.e., $m_{D} X_{\nu} \widetilde{\nu}_{L}^{\dagger} \widetilde{\nu}^{c}$ and $m_{D} M_{R} \widetilde{\nu}_{L}^{\dagger} \widetilde{N}$, in the sneutrino mass matrix eq. (2.10). Therefore, without large fine-tuning, the single family case can not lift $m_{h}$ sizably and at the same time has a sneutrino LSP around the weak scale with a sufficiently small $\widetilde{\nu}_{L}$ fraction. An extra family of sneutrino, which has Yukawa coupling $Y_{\nu_{2}}$ for definiteness, ${ }^{5}$ is thus introduced.

Now good scenarios can be accommodated. Because $Y_{\nu}$, the third family Yukawa coupling, has approached the IR-fixed point, $Y_{\nu_{2}}$ should be much smaller than it. As a result, $\widetilde{\nu}_{2}^{c}$ and $\widetilde{N}_{2}$ can be around the weak scale. While $m_{\widetilde{\nu}_{L, 2}}^{2}$ is assumed to be properly heavier and hence the lightest sneutrino $\widetilde{N}_{1}$ is dominated by $\widetilde{N}_{2}$ and/or $\widetilde{\nu}_{2}^{c}$. According to the analysis made in the above subsection, a sufficient suppression of the doublet component can be realized via a heavy $m_{\widetilde{\nu}_{L, 2}}$ for large $Y_{\nu, 2}$ or a small $Y_{\nu, 2}$ for light $m_{\widetilde{\nu}_{L, 2}}$. Both are well motivated and will be discussed respectively in the following. Hereafter, the subscript "2" will be dropped and sneutrinos refer to the second family unless otherwise specified.

\subsubsection{Higgs-portal sneutrino DM inspired by natural SUSY}

In the natural SUSY framework, the first and second families of sfermions are assumed to be much heavier than the third family, says lying above $3 \mathrm{TeV}$. Such a pattern may be related to the SM fermion flavor structure [45]. While sterile neutrinos are SM singlets and have different flavor structure, and hence their superpartners are allowed to be light. As a result, we naturally get a light sneutrino LSP with negligible $\widetilde{\nu}_{L}$ component.

We are working in the Higgs-portal sneutrino DM, so its correct relic density, viewing from the stringent XENON100 experimental constraint, needs to be studied carefully. As mentioned before, the cross section from DM annihilating into a pair of Higgs bosons via contact interaction is not large enough. Consequently, annihilations of the viable sneutrino

\footnotetext{
${ }^{5}$ As a matter of fact, to produce the realistic neutrino masses and mixings, we need extra families as well.
} 
LSP are completely specified by $h$ in the $s$-channel. Correct relic density restricts the viable sneutrino LSP only to two possibilities (see the left panel of figure 5)

- $m_{\widetilde{N}_{1}} \simeq m_{h} / 2$. When the DM mass lies below the $W$ threshold and closes to the Higgs pole, DM annihilations will benefit from the Higgs resonance enhancement. Then correct relic density can be got for a small $\mu_{h}$. In turn, the XENON100 bound can be evaded, see the right panel of figure 5. The Higgs invisible decay may impose an even more stringent constraint for $m_{\mathrm{DM}}<m_{h} / 2$. In the right panel of figure 5 we label the points giving invisible Higgs decay to a pair of $\widetilde{N}_{1}$ with branching ratio larger than $10 \%$, which, assumed to be the upper bound, excludes $\widetilde{N}_{1}$ below $55 \mathrm{GeV}$.

- $m_{\widetilde{N}_{1}} \gtrsim m_{W}$. Bare in mind that the SM-like Higgs boson decay is always dominated by the $W W$ mode for $m_{h} \gtrsim 160 \mathrm{GeV}$ [46], thus $\widetilde{N}_{1}$ will dominantly annihilate into $W W$ once it is kinematically allowed. Increasing DM mass will decrease the DM annihilation cross section, but new accessible channels such as $Z Z / h h / t \bar{t}$ can partially compensate the decrease. As a result, even without significantly increasing $\mu_{h}$, the sneutrino LSP with mass extending above a few times of $m_{W}$ still can acquire correct relic density, see the left panel of figure 5. On the other hand, a heavier scalar DM helps to reduce $\sigma_{\mathrm{SI}}$ from $h$, so the XENON100 experimental constraint is satisfied for the heavier $\widetilde{N}_{1}$, see figure 5 . Actually, from it we see that the latest LUX result has excluded the sneutrino DM between 65 and $150 \mathrm{GeV}$.

We modify MicrOMEGAs 3.2 [47] by including the ISS-MSSM and then using it to calculate the sneutrino DM relic density and $\sigma_{\mathrm{SI}}$. Based on the previous semi-analytical analysis, we scan the following three-dimensional parameter space

$$
\begin{aligned}
A_{\nu} & =200 \mathrm{GeV}, \quad Y_{\nu} \in[0.05,0.3], \\
m_{\widetilde{N}}^{2} & \in\left[-M_{R}^{2}, M_{R}^{2}\right], \quad m_{\widetilde{\nu}^{c}}^{2}=k m_{\widetilde{N}}^{2} \text { with } k \in[-5.0,5.0] .
\end{aligned}
$$

Additionally, we take $M_{R}=9 m_{D}$ and $B_{M_{R}}=(100 \mathrm{GeV}) M_{R}$, and fix the irrelevant MSSM parameters as the following

$$
\tan \beta=20, \quad m_{\tilde{l}}=10 \mathrm{TeV}, \quad \mu=400 \mathrm{GeV},
$$

while all the other sparticles are decoupled for simplicity. Comments are in orders. First, the upper bound on $Y_{\nu}$ covers the limit indicated by eq. (3.9) and is consistent with the large Yukawa coupling of the third family. Second, we allow negative $m_{\widetilde{\nu}^{c}}^{2}$ and $m_{\widetilde{N}}^{2}$ such that the LSP, via a moderately large cancellation, can be light around $m_{W}$ even for the large $Y_{\nu}$ and heavy $M_{R}$ case. Compared to the method turning back to a large $B_{M_{R}}$ to get a light DM, this way allows a widely varying mixing angle $\theta_{23}$. But they do not show essential difference.

\subsubsection{Coannihilating sneutrino LSP inspired by the semi-constrained ISS}

We now turn to a scenario inspired by the semi-constrained ISS-MSSM. In this scenario, the MSSM part is described by the CMSSM with free parameters $m_{0}, M_{1 / 2}$ and $A_{0}$, etc. 

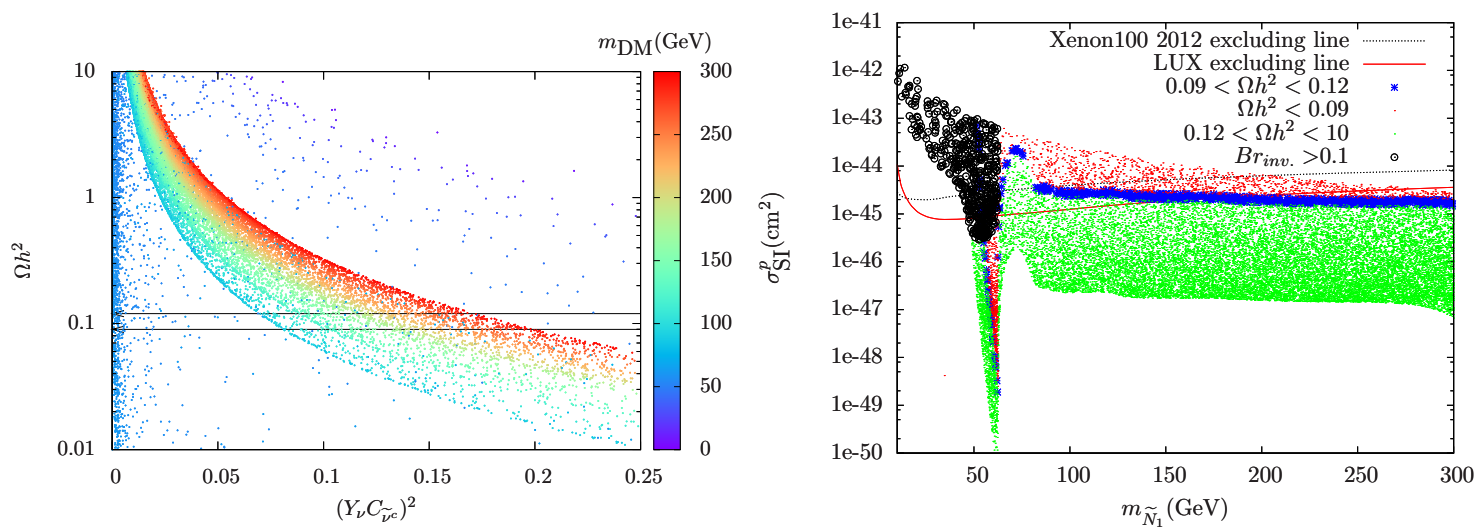

Figure 5. Left: the DM relic density versus the effective coupling constant between the up-type Higgs doublet and sneutrino LSP. Points in the narrow band between the two black lines have relic density consistent with the measurement: $0.09<\Omega h^{2}<0.12$. Right: the Direct detection exclusion limits on the sneutrino LSP in the $\sigma_{\mathrm{SI}}^{p}-m_{\mathrm{DM}}$ plane. Assuming the observed DM relic density can be realized from non-thermal production and dilution mechanism, we also show the points with smaller and larger relic densities, labeled as the red and green points, respectively. Black circles denote the points with branching ratio of Higgs invisible decay $\geq 10 \%$.

The ISS-sector contains the universal SUSY breaking soft mass terms $m_{\widetilde{S}}, A_{\nu}$ and $B_{M_{R}}$. For the sake of enhancing Higgs boson mass, $\left|A_{\nu}\right|$ typically is multi-TeV, says $5 \mathrm{TeV}$ (But the enhancement is purely due to the mixing effect and thus is limited, typically around $2 \mathrm{GeV}$.). Note that $m_{\widetilde{\nu}_{L}}$, by naturalness, is favored to be at the sub-TeV scale. So we need a rather small $Y_{\nu}$, typically at the order of 0.001 , to control $C_{\widetilde{\nu}_{L}}$. The LSP annihilation rate is suppressed by small $Y_{\nu}$, and then the coannihilation effect [48] is needed to reduce its number density.

If the next-to-the LSP (NLSP) and the sneutrino LSP have sufficiently small mass difference $\delta m$, coannihilation effect will play an important role in reducing number density of the sneutrino LSP. The effective annihilation cross section $\sigma_{\text {eff }}$, in terms of ref. [48], is a weighted sum of the LSP-LSP, LSP-NLSP, and NLSP-NLSP annihilation cross sections, denoted as $\sigma_{11}, \sigma_{12}$, and $\sigma_{22}$, respectively. Viewing from the sneutrino system in the scenarios under consideration, a large $\sigma_{\text {eff }}$ should be ascribed to a large $\sigma_{22}$ rather than $\sigma_{12}$. The natural candidates for the NLSP include the Higgsino and doublet-like snerutrino which have full $\mathrm{SU}(2)_{L}$ interactions. While the colored sparticles, especially the light stop, should be moderately heavy owing to the LHC bounds. So we do not consider them in this paper.

We focus on the Higgsino NLSP case. This is a reasonable choice because by naturalness the $\mu$-term should be small, and thus light Higgsinos. We now make a numerical analysis. In terms of the above arguments, we scan a slice of the parameter space as the following

$$
\begin{aligned}
A_{\nu} & =5.0 \mathrm{TeV}, \quad Y_{\nu} \in[0.001,0.01], \quad \mu \in[100,400] \mathrm{GeV} \\
m_{\widetilde{\nu}^{c}} & =m_{\widetilde{N}} \in[\mu-30, \mu+30], \quad m_{\widetilde{l}}=[400,800] \mathrm{GeV} .
\end{aligned}
$$



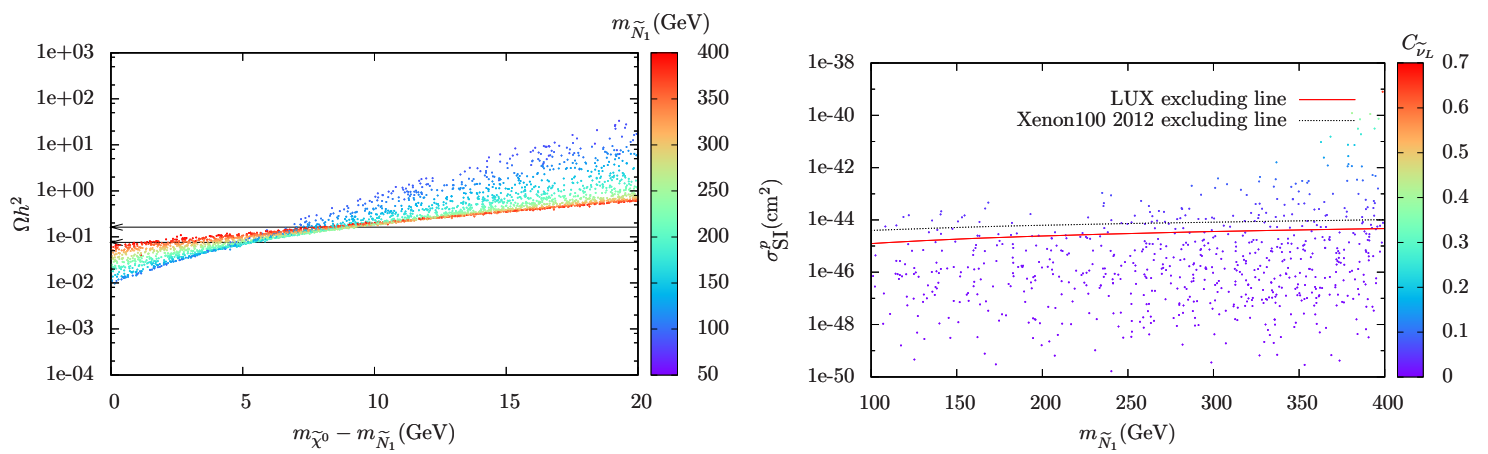

Figure 6. Left: the DM relic density versus the sneutrino-Higgsino mass difference. Right: the direct detection exclusion limits on the sneutrino LSP in the $\sigma_{\mathrm{SI}}^{p}-m_{\mathrm{DM}}$ plane. The heavier LSP may have a larger $C_{\widetilde{\nu}_{L}}$, so it is more sensitive to direct detections.

The other parameters are chosen as before. In this scenario the bounds from direct detections are weak, see the right panel of figure 6 where most of the region is untouched, except for those with a larger $C_{\widetilde{\nu}_{L}}$. This is not surprising. Because $\sigma_{11}$ is allowed to be small, the sneutrino LSP can couple to Higgs, more widely, the visible sector, weakly. As a consequence, it may become deeply dark, even for the indirect detections. Instead, we may have to count on the possible hints if a light non-LSP Higgsino is discovered at colliders.

\section{Discussions and conclusion}

The discovery of a relatively heavy SM-like Higgs boson is a good news to SUSY but not to the MSSM, whose little hierarchy problem is exacerbated. Moreover, the viable parameter space for the neutralino LSP dark matter is greatly constrained. In this work we studied the two aspects of the MSSM extended by the inverse seesaw mechanism, which is an elegant mechanism to realize the low-scale seesaw mechanism without turning to very small Yukawa couplings. This feature makes the model contribute to a sizable radiative correction to $m_{h}$, up to $\sim 4 \mathrm{GeV}$ in the case of an IR-fixed point of the coupling $Y_{\nu} L H_{u} \nu^{c}$ and a large sneutrino mixing. Thus, the little hierarchy problem can be alleviated. Furthermore, it makes the lightest (highly complex) sneutrino be a viable thermal DM candidate. Owing to the stringent constraints from the correct DM relic density and XENON100 experiment, we found that there are only two viable scenarios for the LSP sneutrino

- Its dynamics is reduced to that of the Higgs-portal complex DM, with mass around $m_{h} / 2$ or above $m_{W}$. The upcoming experiments such as XENON1T can exclude them, especially the latter. Additionally, we may observe a hint of DM with resonant enhancement from Higgs invisible decay.

- It is a coannihilating DM, likely with Higgsinos. Because now a fairly weak coupling between the sneutrino DM and visible particles is allowed, it is hard to be excluded.

Taking into account the requirement of enhancing $m_{h}$ which needs large sneutrino mixing, we should introduce an extra family of sneutrinos to account for sneutrino DM. And 
both scenarios naturally work when we attempted to suppress the DM left-handed sneutrino component.

In this article we did not consider the supersymmetric ISS models extended by gauge groups $[33,34,49,50]$. Actually, such supersymmetric models have even more significant effects to enhance the SM-like Higgs boson mass $[49,50]$. The LHC detection in the presence of a singlet-like sneutrino DM possesses some special signatures when the ordinary LSP is a stau sneutrino [51]. Consequently, the colored sparticle like stop decay is characterized by a long decay chain and the presence of leptons in the final state, which may weaken the ATLAS MET plus jets constraint. Other collider phenomeplogy consequences of this model are also studied [52].

\section{Acknowledgments}

We would like to thank Tai Li and Valentina De Romeri for helpful discussions. This research was supported in part by the China Postdoctoral Science Foundation (No. 2012M521136) (ZK), by the Natural Science Foundation of China under grant numbers 10821504, 11075194, 11135003, and 11275246 (JG, TL, YL), and by the National Basic Research Program of China (973 Program) under grant number 2010CB833000.

Open Access. This article is distributed under the terms of the Creative Commons Attribution License (CC-BY 4.0), which permits any use, distribution and reproduction in any medium, provided the original author(s) and source are credited.

\section{References}

[1] ATLAS collaboration, Observation of a new particle in the search for the Standard Model Higgs boson with the ATLAS detector at the LHC, Phys. Lett. B 716 (2012) 1 [arXiv: 1207.7214] [INSPIRE].

[2] CMS collaboration, Observation of a new boson at a mass of $125 \mathrm{GeV}$ with the CMS experiment at the LHC, Phys. Lett. B 716 (2012) 30 [arXiv:1207.7235] [INSPIRE].

[3] G. Jungman, M. Kamionkowski and K. Griest, Supersymmetric dark matter, Phys. Rept. 267 (1996) 195 [hep-ph/9506380] [INSPIRE].

[4] R. Mohapatra and J. Valle, Neutrino Mass and Baryon Number Nonconservation in Superstring Models, Phys. Rev. D 34 (1986) 1642 [INSPIRE].

[5] M. Gonzalez-Garcia and J. Valle, Fast Decaying Neutrinos and Observable Flavor Violation in a New Class of Majoron Models, Phys. Lett. B 216 (1989) 360 [INSPIRE].

[6] Z. Kang, J. Li and T. Li, On naturalness of the MSSM and NMSSM, JHEP 11 (2012) 024 [arXiv: 1201.5305] [INSPIRE].

[7] H. Baer, V. Barger and A. Mustafayev, Implications of a $125 \mathrm{GeV}$ Higgs scalar for LHC SUSY and neutralino dark matter searches, Phys. Rev. D 85 (2012) 075010 [arXiv: 1112.3017] [INSPIRE].

[8] M. Kadastik, K. Kannike, A. Racioppi and M. Raidal, Implications of the $125 \mathrm{GeV}$ Higgs boson for scalar dark matter and for the CMSSM phenomenology, JHEP 05 (2012) 061 [arXiv:1112.3647] [INSPIRE]. 
[9] J. Cao, Z. Heng, D. Li and J.M. Yang, Current experimental constraints on the lightest Higgs boson mass in the constrained MSSM, Phys. Lett. B 710 (2012) 665 [arXiv:1112.4391] [INSPIRE].

[10] M. Perelstein and B. Shakya, XENON100 Implications for Naturalness in the MSSM, NMSSM and lambda-SUSY, Phys. Rev. D 88 (2013) 075003 [arXiv:1208.0833] [InSPIRE].

[11] C. Boehm, P.S.B. Dev, A. Mazumdar and E. Pukartas, Naturalness of Light Neutralino Dark Matter in pMSSM after LHC, XENON100 and Planck Data, JHEP 06 (2013) 113 [arXiv:1303.5386] [INSPIRE].

[12] H.E. Haber and R. Hempfling, The renormalization group improved Higgs sector of the minimal supersymmetric model, Phys. Rev. D 48 (1993) 4280 [hep-ph/9307201] [INSPIRE].

[13] M.S. Carena, M. Quirós and C. Wagner, Effective potential methods and the Higgs mass spectrum in the MSSM, Nucl. Phys. B 461 (1996) 407 [hep-ph/9508343] [INSPIRE].

[14] L.E. Ibáñez, The Scalar Neutrinos as the Lightest Supersymmetric Particles and Cosmology, Phys. Lett. B 137 (1984) 160 [inSPIRE].

[15] J.S. Hagelin, G.L. Kane and S. Raby, Perhaps Scalar Neutrinos Are the Lightest Supersymmetric Partners, Nucl. Phys. B 241 (1984) 638 [inSPIRE].

[16] L.J. Hall, T. Moroi and H. Murayama, Sneutrino cold dark matter with lepton number violation, Phys. Lett. B 424 (1998) 305 [hep-ph/9712515] [INSPIRE].

[17] D.G. Cerdeno, C. Muñoz and O. Seto, Right-handed sneutrino as thermal dark matter, Phys. Rev. D 79 (2009) 023510 [arXiv:0807.3029] [INSPIRE].

[18] XENON100 collaboration, E. Aprile et al., Dark Matter Results from 225 Live Days of XENON100 Data, Phys. Rev. Lett. 109 (2012) 181301 [arXiv:1207.5988] [INSPIRE].

[19] LUX collaboration, D. Akerib et al., First results from the LUX dark matter experiment at the Sanford Underground Research Facility, arXiv:1310.8214 [INSPIRE].

[20] M. Hirsch, F. Joaquim and A. Vicente, Constrained SUSY seesaws with a $125 \mathrm{GeV}$ Higgs, JHEP 11 (2012) 105 [arXiv:1207.6635] [INSPIRE].

[21] J. Cao and J.M. Yang, Lightest Higgs boson mass in split supersymmetry with the seesaw mechanism, Phys. Rev. D 71 (2005) 111701 [hep-ph/0412315] [INSPIRE].

[22] Z. Kang, Y. Liu and G.-Z. Ning, Highlights of Supersymmetric Hypercharge \pm 1 Triplets, JHEP 09 (2013) 091 [arXiv:1301.2204] [INSPIRE].

[23] F. Deppisch and J. Valle, Enhanced lepton flavor violation in the supersymmetric inverse seesaw model, Phys. Rev. D 72 (2005) 036001 [hep-ph/0406040] [INSPIRE].

[24] S. Antusch, C. Biggio, E. Fernandez-Martinez, M. Gavela and J. Lopez-Pavon, Unitarity of the Leptonic Mixing Matrix, JHEP 10 (2006) 084 [hep-ph/0607020] [INSPIRE].

[25] S.P. Martin, Extra vector-like matter and the lightest Higgs scalar boson mass in low-energy supersymmetry, Phys. Rev. D 81 (2010) 035004 [arXiv:0910.2732] [InSPIRE].

[26] S.R. Coleman and E.J. Weinberg, Radiative Corrections as the Origin of Spontaneous Symmetry Breaking, Phys. Rev. D 7 (1973) 1888 [InSPIRE].

[27] T. Falk, K.A. Olive and M. Srednicki, Heavy sneutrinos as dark matter, Phys. Lett. B 339 (1994) 248 [hep-ph/9409270] [INSPIRE]. 
[28] C. Arina and N. Fornengo, Sneutrino cold dark matter, a new analysis: Relic abundance and detection rates, JHEP 11 (2007) 029 [arXiv:0709.4477] [INSPIRE].

[29] S. Banerjee, P.S.B. Dev, S. Mondal, B. Mukhopadhyaya and S. Roy, Invisible Higgs Decay in a Supersymmetric Inverse Seesaw Model with Light Sneutrino Dark Matter, JHEP 10 (2013) 221 [arXiv: 1306.2143] [INSPIRE].

[30] J. March-Russell, C. McCabe and M. McCullough, Neutrino-Flavoured Sneutrino Dark Matter, JHEP 03 (2010) 108 [arXiv: 0911.4489] [INSPIRE].

[31] X. Gao, Z. Kang and T. Li, Origins of the Isospin Violation of Dark Matter Interactions, JCAP 01 (2013) 021 [arXiv:1107.3529] [INSPIRE].

[32] K. Huitu, J. Laamanen, L. Leinonen, S.K. Rai and T. Ruppell, Comparison of neutralino and sneutrino dark matter in a model with spontaneous CP-violation, JHEP 11 (2012) 129 [arXiv: 1209.6302] [INSPIRE].

[33] H. An, P.B. Dev, Y. Cai and R. Mohapatra, Sneutrino Dark Matter in Gauged Inverse Seesaw Models for Neutrinos, Phys. Rev. Lett. 108 (2012) 081806 [arXiv:1110.1366] [INSPIRE].

[34] P. Bhupal Dev, S. Mondal, B. Mukhopadhyaya and S. Roy, Phenomenology of Light Sneutrino Dark Matter in cMSSM/mSUGRA with Inverse Seesaw, JHEP 09 (2012) 110 [arXiv:1207.6542] [INSPIRE].

[35] Z. Kang, J. Li, T. Li, T. Liu and J. Yang, Asymmetric Sneutrino Dark Matter in the NMSSM with Minimal Inverse Seesaw, arXiv:1102.5644 [INSPIRE].

[36] N. Arkani-Hamed, L.J. Hall, H. Murayama, D. Tucker-Smith and N. Weiner, Small neutrino masses from supersymmetry breaking, Phys. Rev. D 64 (2001) 115011 [hep-ph/0006312] [INSPIRE].

[37] F. Borzumati and Y. Nomura, Low scale seesaw mechanisms for light neutrinos, Phys. Rev. D 64 (2001) 053005 [hep-ph/0007018] [INSPIRE].

[38] T. Asaka, K. Ishiwata and T. Moroi, Right-handed sneutrino as cold dark matter, Phys. Rev. D 73 (2006) 051301 [hep-ph/0512118] [INSPIRE].

[39] G. Bélanger, M. Kakizaki, E. Park, S. Kraml and A. Pukhov, Light mixed sneutrinos as thermal dark matter, JCAP 11 (2010) 017 [arXiv: 1008.0580] [INSPIRE].

[40] B. Dumont, G. Bélanger, S. Fichet, S. Kraml and T. Schwetz, Mixed sneutrino dark matter in light of the 2011 XENON and LHC results, JCAP 09 (2012) 013 [arXiv:1206.1521] [INSPIRE].

[41] P. Bandyopadhyay, E.J. Chun and J.-C. Park, Right-handed sneutrino dark matter in U(1)' seesaw models and its signatures at the LHC, JHEP 06 (2011) 129 [arXiv:1105.1652] [INSPIRE].

[42] K.-Y. Choi and O. Seto, Light Dirac right-handed sneutrino dark matter, Phys. Rev. D 88 (2013) 035005 [arXiv: 1305.4322] [INSPIRE].

[43] V. De Romeri and M. Hirsch, Sneutrino Dark Matter in Low-scale Seesaw Scenarios, JHEP 12 (2012) 106 [arXiv:1209.3891] [INSPIRE].

[44] C. Arina, F. Bazzocchi, N. Fornengo, J. Romao and J. Valle, Minimal supergravity sneutrino dark matter and inverse seesaw neutrino masses, Phys. Rev. Lett. 101 (2008) 161802 [arXiv:0806.3225] [INSPIRE]. 
[45] A.E. Nelson and D. Wright, Horizontal, anomalous U(1) symmetry for the more minimal supersymmetric standard model, Phys. Rev. D 56 (1997) 1598 [hep-ph/9702359] [INSPIRE].

[46] A. Djouadi, The anatomy of electro-weak symmetry breaking. I: The Higgs boson in the standard model, Phys. Rept. 457 (2008) 1 [hep-ph/0503172] [INSPIRE].

[47] G. Bélanger, F. Boudjema, A. Pukhov and A. Semenov, MicrOMEGAs3.1: a program for calculating dark matter observables, arXiv:1305.0237 [INSPIRE].

[48] K. Griest and D. Seckel, Three exceptions in the calculation of relic abundances, Phys. Rev. D 43 (1991) 3191 [INSPIRE].

[49] A. Elsayed, S. Khalil and S. Moretti, Higgs Mass Corrections in the SUSY B-L Model with Inverse Seesaw, Phys. Lett. B $\mathbf{7 1 5}$ (2012) 208 [arXiv:1106.2130] [INSPIRE].

[50] M. Hirsch, M. Malinsky, W. Porod, L. Reichert and F. Staub, Hefty MSSM-like light Higgs in extended gauge models, JHEP 02 (2012) 084 [arXiv: 1110.3037] [INSPIRE].

[51] S. Mondal, S. Biswas, P. Ghosh and S. Roy, Exploring novel correlations in trilepton channels at the LHC for the minimal supersymmetric inverse seesaw model, JHEP 05 (2012) 134 [arXiv:1201.1556] [INSPIRE].

[52] A. Das and N. Okada, Inverse Seesaw Neutrino Signatures at LHC and ILC, Phys. Rev. D 88 (2013) 113001 [arXiv:1207.3734] [INSPIRE].

[53] I. Gogoladze, B. He and Q. Shafi, Inverse Seesaw in NMSSM and 126 GeV Higgs Boson, Phys. Lett. B 718 (2013) 1008 [arXiv: 1209.5984] [INSPIRE]. 